\title{
Encapsulation of Paclitaxel with Glycol Chitosan Nanoaggregates for in vitro Drug Delivery
}

J. Choi**, A. Calabro*, C. Queenan*, D. Becker*, D. Leonardi**

* Bergen County Academies, Nano-Structural Imaging Lab, 200 Hackensack Avenue, Hackensack, NJ 07601

** Bergen County Academies, Biotechnology Lab, 200 Hackensack Avenue, Hackensack, NJ 07601

Paclitaxel (PTX) is a chemotherapeutic agent that induces apoptosis by binding to Bcl-2 (apoptosis stopping protein) and the $\beta$-subunit of tubulin. PTX is commonly delivered with Cremophor-EL solvent in an inclusion complex called Taxol, which has been shown to cause severe hypersensitivity reactions in chemotherapy patients. In this study, self-assembled nanoparticles were developed as novel excipients to minimize the cytotoxicity of PTX. Fluorescent glycol-chitosan nanoaggregate (GCF) drug carriers were engineered and PTX was incorporated to form a GCF-PTX conjugate [1]. The conjugates, Taxol inclusion complex and Cremophor-EL solvent were all tested for their effects on MDA-MB-231 human breast cancer cells and CRL-1502 human dermal fibroblast cells.

To analyze the successful creation of the conjugates, $3 \mu 1$ aliquots each of GCF-PTX and GCF were placed onto 200-mesh copper grids for imaging by transmission electron microscopy (TEM) with a $200 \mathrm{kV}$ JEOL JEM-2100 (Figure 1). MDA-MB-231 cells were examined by fluorescent microscopy to observe the endocytosis of GCF (Figure 2). Six-well chamber slides were prepared at a density of 20,000 cells per chamber. The slides were incubated for 24 hours at $37^{\circ} \mathrm{C}$ and $5 \% \mathrm{CO}_{2}$, treated with GCF or GCF-PTX, and again incubated for 24 hours. Chambers were removed and slides were rinsed in phosphate buffered saline. Three drops of ProLong Gold Antifade Reagent ${ }^{\circledR}$ were added to the slides for coverslip mounting and cured for 24 hours in the dark. The slides were examined using a Nikon 80i Fluorescent Microscope with FITC filters (excitation/emission 490nm/520nm).

Cell viability testing showed that Cremophor-based treatments were extremely cytotoxic $(p<0.001)$. When compared to the Cremophor-based treatments, GCF-PTX-induced apoptosis in MDA cells was significantly reduced $(\mathrm{p}<0.001)$. Empty GCF nanoparticle sample groups were examined as a control and showed no statistically significant apoptosis. As a result, using a nanoparticle excipient improved the efficacy of targeted drug delivery by reducing cytotoxicity.

References

[1] M. Lee, et al., Colloid Polymer Science. 284 (2006) 506-512.

[2] The authors would like to acknowledge the administration of the Bergen County Technical Schools for their continued support of the research program. 

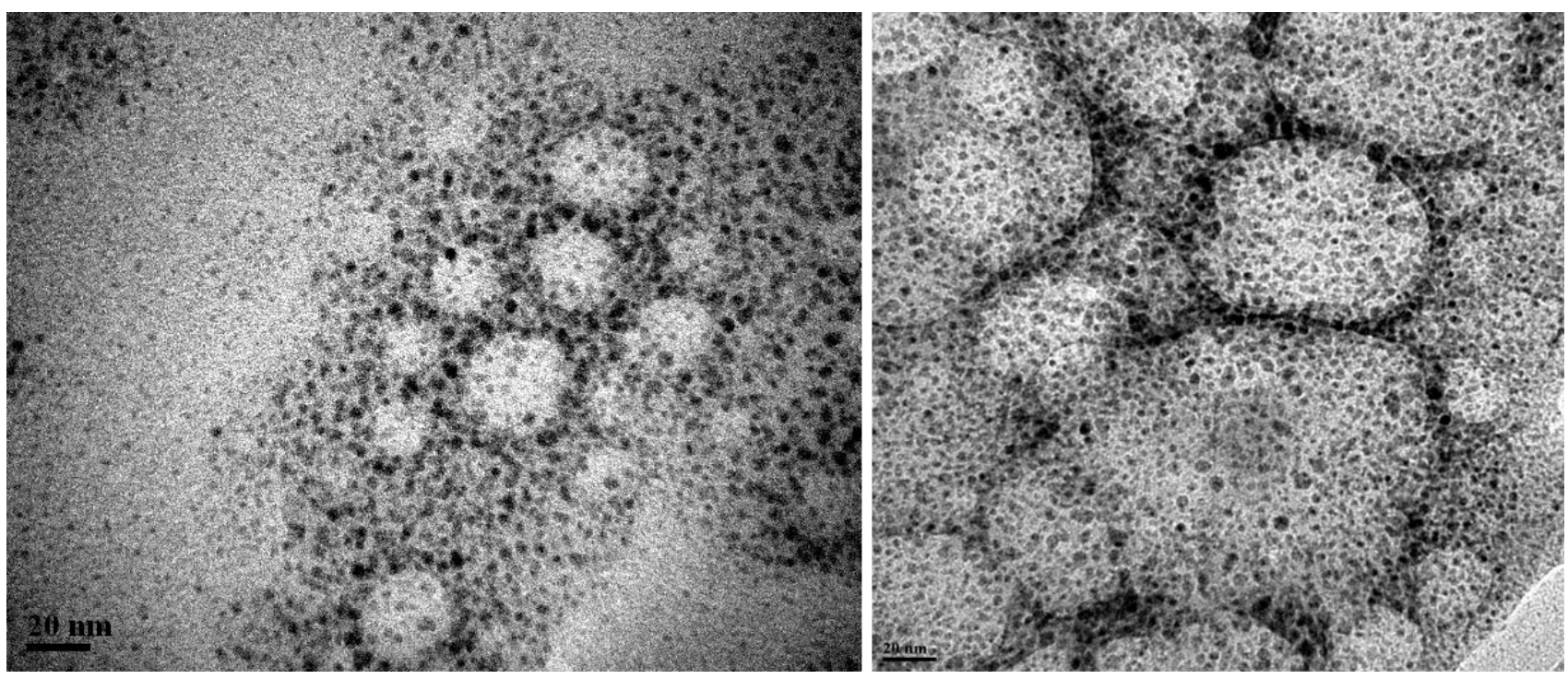

Figure 1. TEM micrographs of GCF-PTX self-conjugating nanoparticles. The diameter for each particle (distinct, circular regions on the micrographs) was found to be approximately 60nm (scale bars $=20 \mathrm{~nm}$ ). Each cluster of nanoparticles had a diameter of approximately $200 \mathrm{~nm}$.

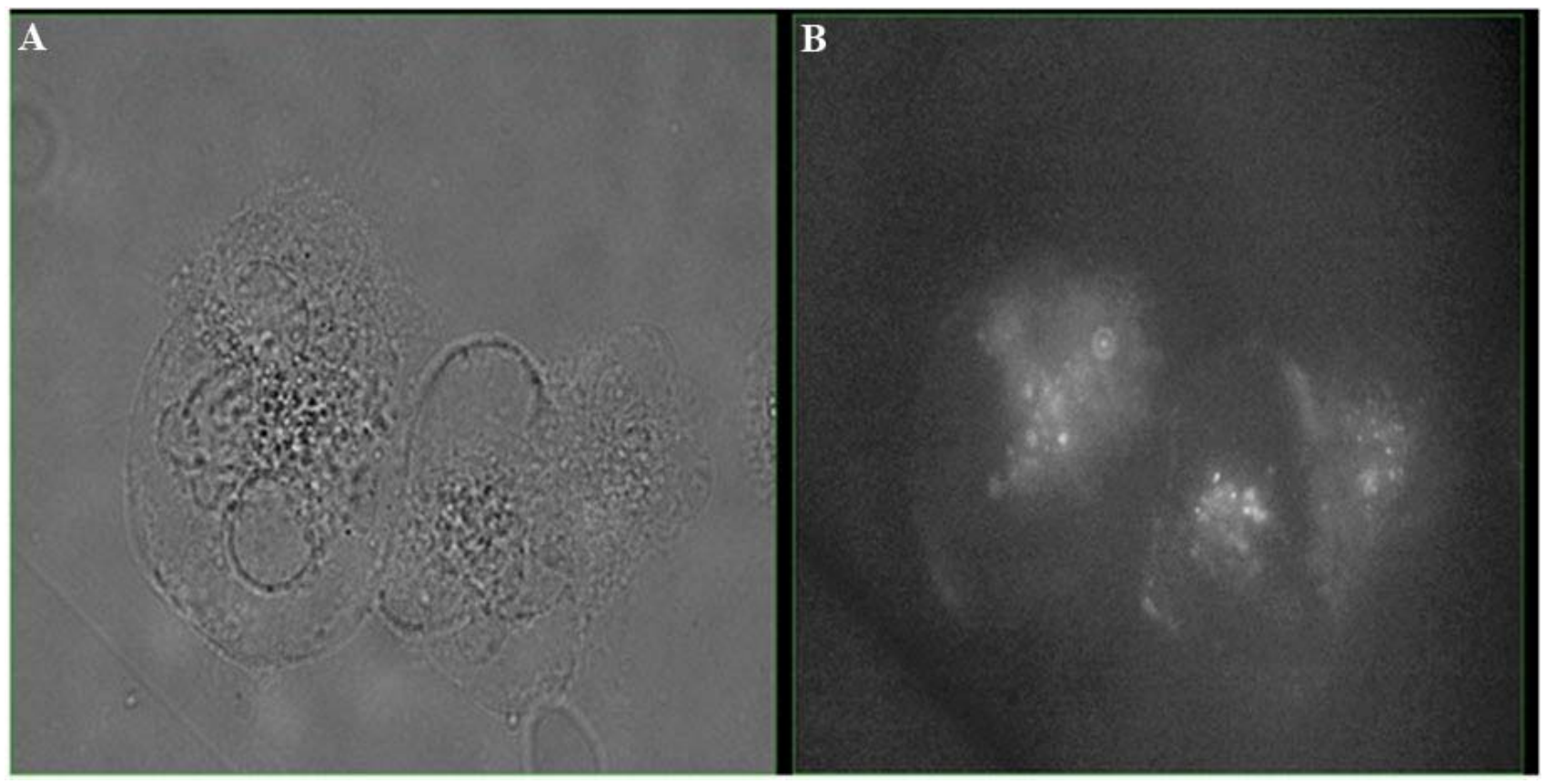

Figure 2. Light microscopy images of GCF-PTX nanoparticle cellular uptake. (A) Bright field image of MDA-MB-231 cells at 1000X. (B) Fluorescent image (excitation/emission 490nm/520nm) of the same sample. Endocytosis of fluorescent nanoparticles in the MDA-MB-231 cells was evident by white areas within the cells. 